\title{
Future lunar mission Active X-ray Spectrometer development: Surface roughness and geometry studies
}

\author{
M. Naito ${ }^{\text {a,*, N. Hasebe }}{ }^{\text {a,b }}$, H. Kusano ${ }^{\mathrm{b}}$, H. Nagaoka ${ }^{\mathrm{a}, \mathrm{b}}$, M. Kuwako ${ }^{\mathrm{a}}$, Y. Oyama ${ }^{\mathrm{a}}$, \\ E. Shibamura ${ }^{\text {b }}$, Y. Amano ${ }^{\text {b }}$, T. Ohta ${ }^{\text {, }}$, K.J. Kim ${ }^{\text {d }}$, J.A.M. Lopes ${ }^{\text {e,f }}$ \\ a School of Advanced Science and Engineering, Waseda University, 3-4-1 Okubo, Shinjuku, Tokyo, Japan \\ ${ }^{\mathrm{b}}$ Research Institute for Science and Engineering, Waseda University, 3-4-1 Okubo, Shinjuku, Tokyo, Japan \\ ' School of Education and Integrated Arts and Science, Waseda University, 1-104 Totsuka, Shinjuku, Tokyo, Japan \\ ${ }^{\mathrm{d}}$ Korea Institute of Geoscience and Mineral Resources, Gwahang-no 124, Yuseong-gu, Daejeon 305-350, South Korea \\ e Department of Physics, University of Coimbra, Apartado 3020, 3001-401 Coimbra, Portugal \\ ${ }^{\mathrm{f}}$ Instituto Superior de Engenharia de Coimbra, 3030-199 Coimbra, Portugal
}

\section{A R T I C L E I N F O}

\section{Article history:}

Received 27 November 2014

Received in revised form

2 March 2015

Accepted 29 March 2015

Available online 7 April 2015

Keywords:

The moon

$\mathrm{X}$-ray fluorescence

Active X-ray Spectrometer

\begin{abstract}
A B S T R A C T
The Active X-ray Spectrometer (AXS) is considered as one of the scientific payload candidates for a future Japanese mission, SELENE-2. The AXS consists of pyroelectric X-ray generators and a Silicon Drift Detector to conduct X-Ray Fluorescence spectroscopy (XRF) on the Moon to measure major elements: $\mathrm{Mg}, \mathrm{Al}, \mathrm{Si}, \mathrm{Ca}$, Ti, and Fe; minor elements: $\mathrm{Na}, \mathrm{K}, \mathrm{P}, \mathrm{S}, \mathrm{Cr}$ and $\mathrm{Mn}$; and the trace element Ni depending on their concentration. Some factors such as roughness, grain size and porosity of sample, and the geometry of X-ray incidence, emission and energy will affect the XRF measurements precision. Basic studies on the XRF are required to develop the AXS. In this study, fused samples were used to make homogeneous samples free from the effect of grain size and porosity. Experimental and numerical studies on the XRF were conducted to evaluate the effects from incidence and emission angles and surface roughness. Angle geometry and surface roughness will be optimized for the design of the AXS on future missions from the results of the experiment and the numerical simulation.
\end{abstract}

(c) 2015 Elsevier B.V. All rights reserved.

\section{Introduction}

Determining the distribution of elements in the lunar surface is essential for lunar science as it characterizes the geochemistry, and also supports the understanding of geology, of lunar surface materials. Great progress on lunar science has been achieved so far by recent observations made by lunar orbiters such as the Lunar Prospector [1], Clementine [2], SELENE (Kaguya) [3], Chang'E-1 [4] and -2 [5], and Chandrayaan-1 [6]. A landing mission will provide more detailed information on the local landing site area, improving the level of information on the Moon obtained by the previous lunar orbiters. In Japan, SELENE-2 is being developed as a follow-on mission of the first lunar orbiter of SELENE (Kaguya). SELENE-2 is a landing mission with a roving vehicle [7].

An Active X-ray Spectrometer (AXS) [8], one of the scientific payload candidates on the rover, is an in situ element composition analyzer for SELENE-2. It will be mounted on the arm head of the

\footnotetext{
* Corresponding author. Tel./fax: +8135286 3897.

E-mail address: com-nm@akane.waseda.jp (M. Naito).
}

lunar rover of the SELENE-2 mission together with a Rock Abrasion Tool (RAT). The AXS will determine elemental concentration of various samples: various kinds of rocks and regolith samples at the landing site and along the path of the rover. The AXS measures lunar major elements: $\mathrm{Mg}, \mathrm{Al}, \mathrm{Si}, \mathrm{Ca}, \mathrm{Ti}$, and $\mathrm{Fe}$; minor elements: $\mathrm{Na}, \mathrm{K}, \mathrm{P}, \mathrm{S}, \mathrm{Cr}$ and $\mathrm{Mn}$.

The AXS consists of pyroelectric X-ray generators and a Silicon Drift Detector (SDD) [8]. The AXS performs X-Ray Fluorescence spectroscopy (XRF) to determine elemental composition. The XRF is a well established laboratory technique. In general, an X-ray tube is used as an X-ray source for XRF in laboratory experiments. However, the X-ray tube requires high voltage supply significantly increasing the payload weight. In space missions, the XRF instrument should be light in weight and low in electric power consumption due to the severe restrictions on the payload resources. All the XRF instruments carried on previous lunar and Mars missions such as Chang'E-3 [9], Viking [10], Mars Pathfinder [11], MER [12], and MSL [13] used radioisotopes such as ${ }^{55} \mathrm{Fe},{ }^{109} \mathrm{Cd}$, and ${ }^{244} \mathrm{Cm}$ to excite planetary surface material elements. Although the use of those radioisotopes has extraordinary advantage of light weight and no need of high voltage supply, it is not allowed at present for Japanese missions to 
bring or send radioisotope sources. There are some alternatives to the radioisotope sources, and the X-ray generator using pyroelectric crystals is one of them [14-16].

Features of the pyroelectric X-ray generator are small size, light weight, low power consumption, no high voltage power supply, and X-ray emission only when needed. Therefore, the AXS is suitable for a Japanese roving mission on the Moon.

Samples for laboratory XRF measurements are finely ground to smooth surfaces, whereas planetary surface is contaminated by micro-meteoroids and other cosmic factors. The weathering effect makes analysis complex. In order to reduce the weathering effect, the uppermost sample surface will be removed by the RAT. Moreover, there are some factors such as sample surface roughness, grain size and porosity, geometry of X-ray incidence, and emission and energy, which might affect the precision of XRF measurements [17-20]. Therefore, we have to investigate the effect of those factors.

In our previous study [21], we used fused samples to make homogeneous samples and remove the effect from grain size and porosity of surface and considered the effect of roughness, incidence angle, and emission angle. Various surface roughness of material samples is produced by grinding samples with different hones. In this work, we performed a more detailed measurement related to the surface roughness below $2 \mu \mathrm{m}$, and numerical calculations to simulate the experiments. The experiments in this work provide important information to design the configuration of $\mathrm{X}$-ray generator, SDD and sample position in the AXS. The results obtained by the experiments and simulations reported in this work are related with the surface roughness of samples and incidence angle of X-ray.

\section{Methods}

\subsection{Experimental setup}

The experimental setup is described in detail in Ref. [21]. Here, we briefly describe the experimental apparatus and sample preparation.

In this study, the X-ray fluorescence analysis was performed in vacuum at a pressure of about $4 \mathrm{~Pa}$. An X-ray tube with a silver target (Amptek Mini-X Silver (Ag)) was used as an X-ray source, and an SDD (Amptek XR-100SDD) with a detector size of $25 \mathrm{~mm}^{2} \times 500 \mu \mathrm{m}$ and a Be window of $8 \mu \mathrm{m}$ in thickness was used as an X-ray detector. Fig. 1 shows the energy spectrum of Xrays emitted from the Mini-X through a $1 \mathrm{~mm} \varnothing$ hole $\mathrm{Pb}$ collimator

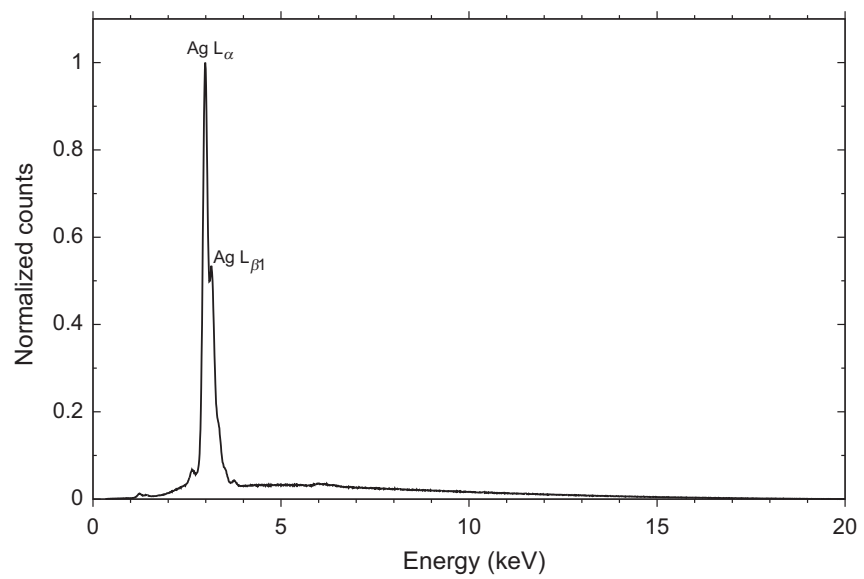

Fig. 1. Energy spectrum of the X-rays emitted from the Mini-X measured by the SDD. A sum peak of $\mathrm{Ag} \mathrm{L}_{\alpha}(2.98 \mathrm{keV})$ and $\mathrm{L}_{\beta 1}(3.15 \mathrm{keV})$ around $3 \mathrm{kev}$, and high energy tail of bremsstrahlung were detected. X-ray counts are normalized at $\mathrm{Ag} \mathrm{L}_{\alpha}$. measured by the SDD. The applied voltage and current to Mini-X were $20 \mathrm{kV}$ and $100 \mu \mathrm{A}$ for all measurements, respectively. The Xray beam was collimated with brass and acrylic collimators. The distance between the collimator and sample surface was $5 \mathrm{~cm}$, and that between the sample surface and SDD was $2.2 \mathrm{~cm}$. In this study, two kinds of angle geometry were investigated as shown in Fig. 2. In the geometry $\mathrm{A}$, the emission angle $\theta_{e}$ was fixed at $0^{\circ}$, thus the sample surface is parallel to the SDD window. In the geometry $\mathrm{B}$, on the other hand, the incidence angle $\theta_{i}=\theta_{e}$. The incidence angle $\theta_{i}$ was set at $15^{\circ}, 30^{\circ}, 45^{\circ}$, and $60^{\circ}$ in each geometry.

The glass samples used in this study were made from the geochemical reference samples of JGb-1 (Gabbro) and JP-1 (Peridotite) [22,23] supplied by National Institute of Advanced Industrial Science and Technology, Japan. The powder of each reference sample was mixed with $\mathrm{Li}_{2} \mathrm{~B}_{4} \mathrm{O}_{7}$ in the weight ratio of $1: 2$ and heated at $1200{ }^{\circ} \mathrm{C}$ to fuse, and then cooled down to glass. The glass samples have the size of $36 \mathrm{~mm} \varnothing \times 3 \mathrm{~mm}$ and density of $1.53 \mathrm{~g} / \mathrm{cm}^{3}$ (JGb-1) and $1.76 \mathrm{~g} / \mathrm{cm}^{3}$ (JP-1). This thickness is much larger than the attenuation length of $\mathrm{Ag} \mathrm{L}_{\alpha}$. The chemical composition of major elements in these samples is shown in Table 1 . The $\mathrm{K}_{\alpha} \mathrm{X}$-ray energy of these elements is also listed. We selected the reference samples of JGb- 1 and JP-1 in order to measure a variety of major elements. The measured elements were $\mathrm{Al}, \mathrm{Si}, \mathrm{Ca}$, and $\mathrm{Fe}$ for JGb-1, and $\mathrm{Mg}$, Si, and Fe for JP-1.

The sample surface was ground by hones to investigate the effect of sample surface roughnesses. We used seven kinds of hones with different mesh numbers, \#100, \#180, \#400, \#800, $\# 1500, \# 3000$, and \#6000, to obtain different surface roughness. The mesh number is related to the grain size on hones, and the sample surface becomes smooth when using hone with large mesh number. Here, the surface roughness is evaluated by the arithmetic average roughness $R_{a}$, which is defined by

$R_{a}=\frac{1}{l} \int_{0}^{l}|h(x)| d x$

where $l$ is the measured length, $x$ the distance from the origin, and $h(x)$ the deviation of surface height from the mean line at the (a)

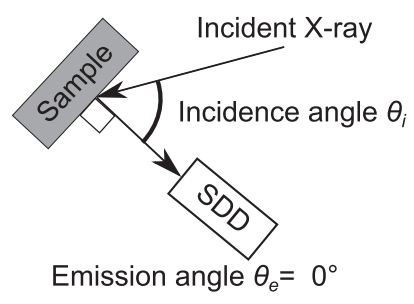

(b)

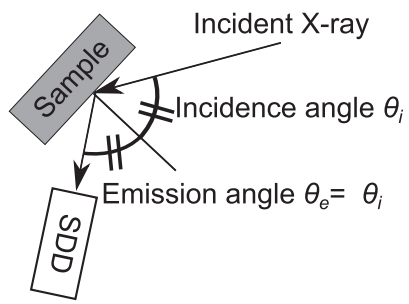

Fig. 2. Schematic drawing of the two angle geometry of experiments: (a) geometry $\mathrm{A}$ and (b) geometry B.

Table 1

The chemical composition of major elements in geochemical reference samples of JGb-1 and JP-1, and the $\mathrm{K}_{\alpha}$ X-ray energy of each element [22,23].

\begin{tabular}{lccl}
\hline Elements & JGb-1 (at\%) & JP-1 (at\%) & $\mathrm{K}_{\alpha}$ X-ray energy $(\mathrm{keV})$ \\
\hline $\mathrm{Mg}$ & 4.15 & 21.6 & 1.25 \\
$\mathrm{Al}$ & 7.31 & 0.25 & 1.49 \\
$\mathrm{Si}$ & 15.5 & 13.8 & 1.74 \\
$\mathrm{Ca}$ & 4.52 & 0.19 & 3.69 \\
$\mathrm{Ti}$ & 0.43 & - & 4.51 \\
$\mathrm{Fe}$ & 4.02 & 2.05 & 6.40 \\
$\mathrm{O}$ & 59.7 & 55.9 & \\
Others & 4.34 & 6.19 & \\
\hline
\end{tabular}




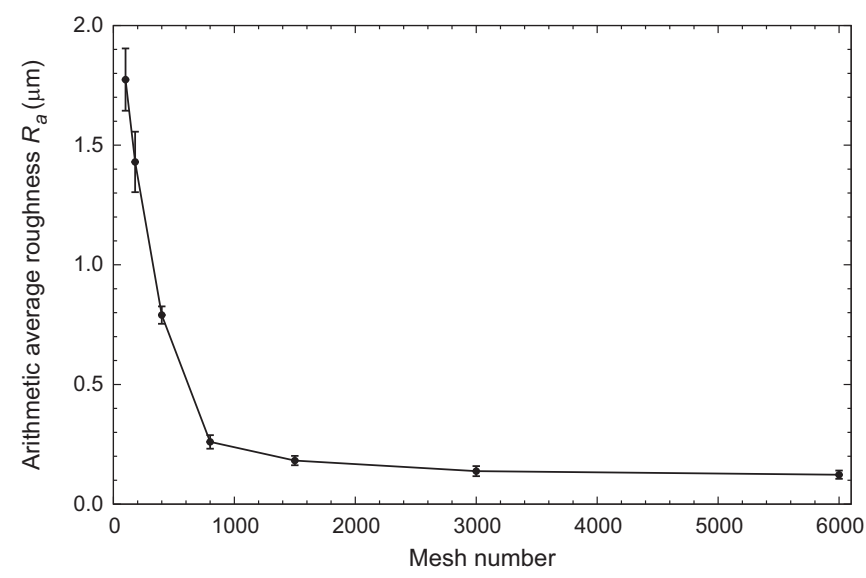

Fig. 3. Relation between the mesh number and arithmetic average roughness $R_{a}$.

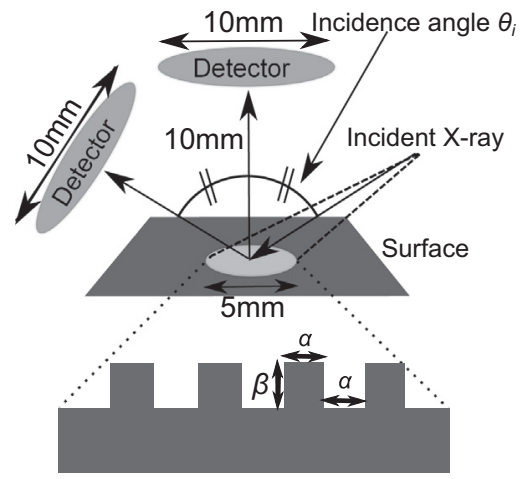

Fig. 4. Schematic drawing of the numerical simulation geometry.

distance $x$. The $R_{a}$ was measured using a surface roughness measuring instrument (KOSAKA Surfcorder SE-3400). Fig. 3 shows the relation between the mesh number and $R_{a}$. The measured length $l$ was about $5 \mathrm{~mm}$ and measuring pin diameter was $2 \mu \mathrm{m}$. The $R_{a}$ represents the average of that for JGb- 1 and JP- 1 , and each $R_{a}$ for JGb- 1 and JP- 1 was determined as the average of measurements of six different points. The error bar represents the measurements of standard deviation. The $R_{a}$ becomes small with the increasing mesh number. However, relation between the mesh number and the $R_{a}$ is not linear and the variation of the $R_{a}$ is small in mesh numbers above $\# 800$.

\subsection{Numerical simulation model and implementation}

The numerical simulation was performed using the Monte Carlo simulation code system PENELOPE version 2008 [24], which can calculate the transport of electrons, positrons, and photons inside a material in an energy range from $50 \mathrm{eV}$ to $1 \mathrm{GeV}$.

Fig. 4 shows the schematic drawing of numerical simulation geometry. The chemical composition of sample was simulated using that of JGb-1 and JP-1 as shown in Table 1 . The sample density was 1.53 and $1.76 \mathrm{~g} / \mathrm{cm}^{3}$ for JGb-1 and JP-1, respectively. To simplify the numerical simulation, the surface roughness was modeled as shown in Fig. 4, which is characterized by the width $\alpha$ and height $\beta$. In this study, the $\alpha$ was fixed at $4 \mu \mathrm{m}$, and the $\beta$ was set at 4,6 , and $8 \mu \mathrm{m}$ to confirm the XRF measurement when the sample surface is rougher than that of the experiments. Then the $R_{a}$ in the numerical simulation is defined as $\beta / 2$. The circular area with a diameter of about $5 \mathrm{~mm}$ at the center of sample surface was irradiated by $5 \times 10^{8}$ counts of $\mathrm{X}$-rays, and then fluorescent X-rays were observed by two detectors with $10 \mathrm{~mm}$ diameter. The distance between sample surface and detector was $10 \mathrm{~mm}$. These two detectors were positioned in the

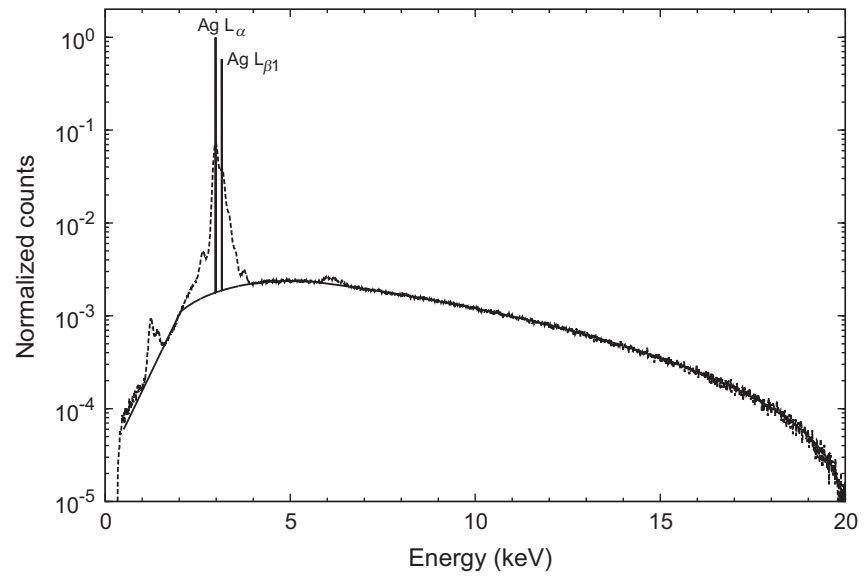

Fig. 5. X-ray energy spectrum for the Mini-X. The solid line shows the input X-ray spectrum for the numerical simulation, and the dotted line shows the X-ray spectrum emitted from the Mini-X measured by the SDD. The peaks around $1.5 \mathrm{keV}$ and $3 \mathrm{keV}$ are an $\mathrm{Al} \mathrm{K} \mathrm{K}_{\alpha}(1.49 \mathrm{keV})$ background from a vacuum chamber and a sum peak of $A g \mathrm{~L}_{\alpha}(2.98 \mathrm{keV})$ and $\mathrm{L}_{\beta 1}(3.15 \mathrm{keV})$. X-ray counts are normalized at Ag $\mathrm{L}_{\alpha}$.

angle geometry corresponding to the geometry A and B in Fig. 2, and the incidence angle $\theta_{i}$ was set at $15^{\circ}, 30^{\circ}, 45^{\circ}$, and $60^{\circ}$. The energy spectrum of incident X-rays was defined to be the emission X-rays from Mini-X as shown in Fig. 1. The incident X-ray spectrum was assumed to be composed of the $\mathrm{Ag} \mathrm{L}_{\alpha}(2.98 \mathrm{keV})$ and $\mathrm{Ag} \mathrm{L}_{\beta 1}$ (3.15 keV) lines with a polynomial continuum background. The total $\mathrm{X}$-ray counts in these two lines were derived from fitting the peak region with Gaussian curves with a polynomial continuum background, and then assigned to the known energy in the incident X-ray spectrum as shown in the solid line in Fig. 5.

\section{Results}

\subsection{Experimental results}

Fig. 6 shows typical energy spectra of fluorescent X-rays measured for samples of JGb- 1 and JP-1. The measurements were made in geometry $A$ for $720 \mathrm{~s}$ at an incidence angle $\theta_{i}=45^{\circ}$, where the samples were ground by a $\# 800$ hone. Fluorescent X-rays of Fe, $\mathrm{Ca}, \mathrm{Ti}, \mathrm{Si}$, and $\mathrm{Mg}$ are detected from JGb-1 and that of $\mathrm{Fe}, \mathrm{Si}$, and $\mathrm{Al}$ are detected from JP-1. Scattering of the incident X-rays $\left(\mathrm{Ag} \mathrm{L}_{\alpha}\right.$ and $\mathrm{L}_{\beta 1}$ ) is also detected from both JGb-1 and JP-1. By fitting a Gaussian function to each peak in the spectra, we obtained a count number for each $\mathrm{K}_{\alpha}$ line. In the following figures, the error bars indicate statistical uncertainty $( \pm 1 \sigma)$ and the data acquisition time is $720 \mathrm{~s}$.

Fig. 7 shows the ratio of fluorescent X-ray counts of elements for various mesh numbers and a $45^{\circ}$ incidence angle, normalized by the Fe peak count number. The ratios in the following figures are normalized by the average of respective values. We hereafter call the ratio as the relative ratio.

Figs. 8 and 9 show the relative ratios as a function of the incidence angle for geometry A and B.

\subsection{Numerical simulation results}

Fig. 10 shows the numerical simulation results of the count number relative ratio as a function of the roughness parameter $\beta$ at an incidence angle $\theta_{i}=45^{\circ}$.

Fig. 11 shows the intensity of the full energy spectrum of X-rays as a function of emission angle $\theta_{e}$ from JGb- 1 with $\beta=0 \mu \mathrm{m}$, for the same incident X-ray spectra as defined above. The intensity of 
(a)

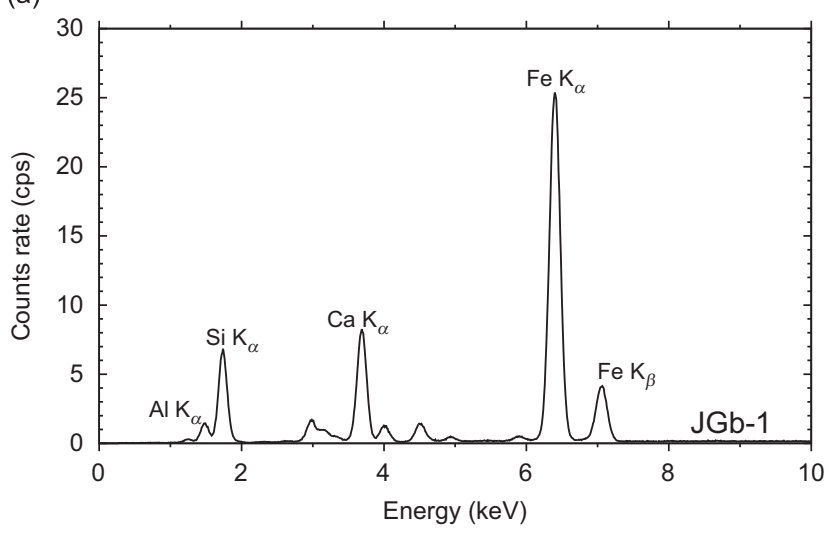

(b)

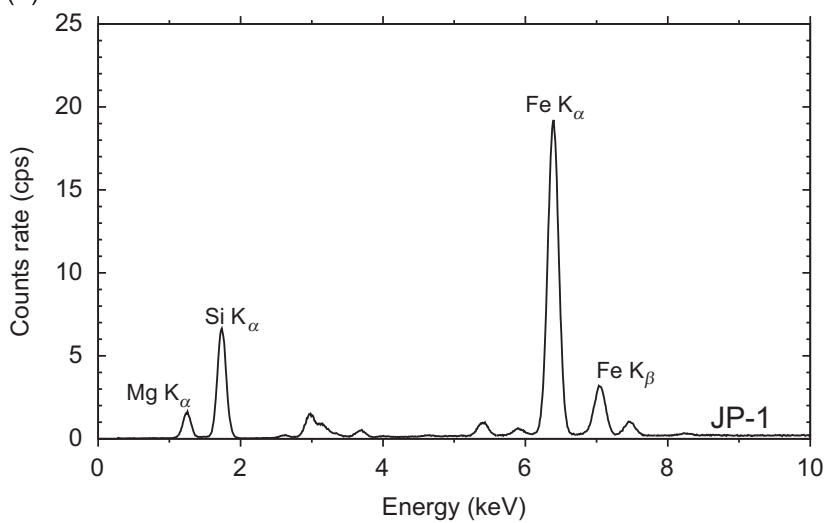

Fig. 6. Energy spectra for JGb-1 (a) and JP-1 (b) ground by a \#800 hone in geometry A at $45^{\circ}$ incident angle.

the fluorescence X-rays is normalized by solid angle and the intensity of the incident X-rays. The numerical simulation with roughness $\beta=4 \mu \mathrm{m}$ has also been carried out. They have been observed to be almost similar to those with roughness $\beta=0 \mu \mathrm{m}$.

\section{Discussion}

\subsection{Dependence on roughness}

As shown in Fig. 3, the variation of $R_{a}$ by hones with mesh numbers over \#800 was very small ( $<8.5 \%$ ). Therefore, grinding by hones with the mesh number $\# 800$ is sufficient enough to grind surface.

Both experimental and simulated relative ratios as a function of roughness are found to be nearly constant. This implies that there is no large correlation between fluorescent X-ray yield and surface roughness at $45^{\circ}$ incidence angle.

\subsection{Dependence on incidence angle}

\subsubsection{Geometry $A$}

Although the distribution of incident X-ray intensity in the irradiated area varies slightly with the incidence angle, it is neglected in the following discussion in order to simplify the model.

As shown in Fig. 8, the relative ratio obtained by the experiment in geometry A becomes larger as the incidence angle increases, and this trend becomes clear in the smooth surface. The variation of the (a)

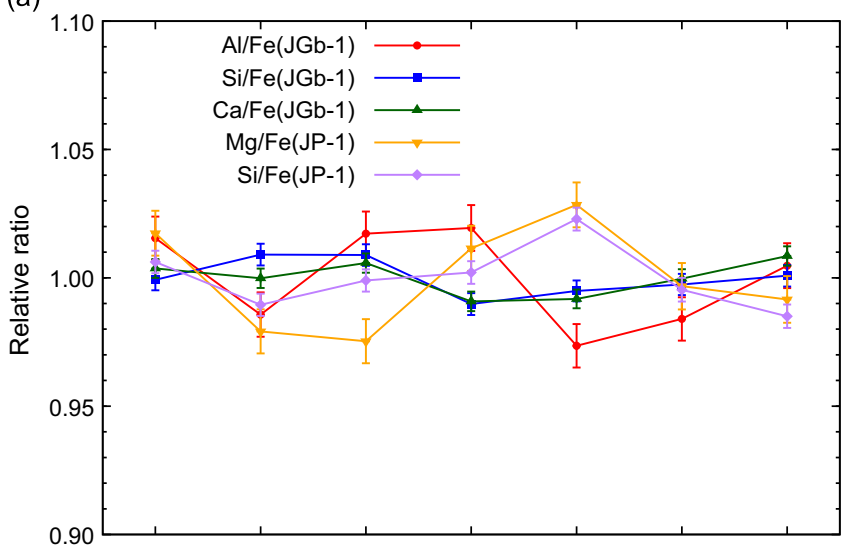

(b)

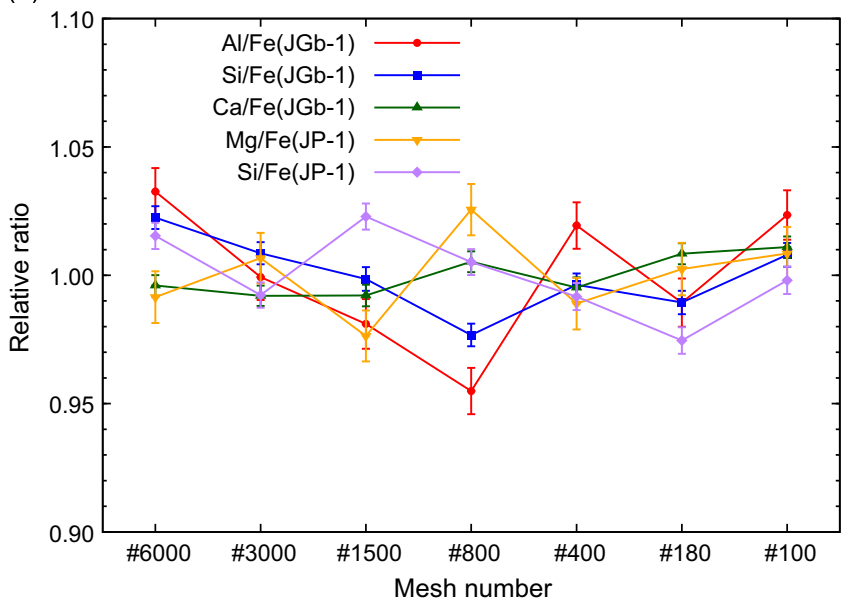

Fig. 7. The count number relative ratios as a function of the mesh numbers at 45 incidence angle. (a) and (b) are obtained in geometry A and B, respectively.

fluorescence X-rays normalized intensity depending on the incidence angle in Fig. 11 also shows similar results. In Fig. 11, the solid angle subtended by the SDD in the geometry A corresponds to integral absorption of the fluorescence X-rays for emission angle below $13^{\circ}$.

As the incidence angle increases, the depth in the sample at which the fluorescence X-rays are created decreases. Low energy $\mathrm{X}$-rays have very small attenuation length, only a few $\mu \mathrm{m}$, which means a significant increase in the X-ray fluorescence intensity escaping from the sample as incidence angle rises. On the other hand, the fact that higher energy X-rays have larger attenuation lengths makes this effect less significant. Note that attenuation lengths of $\mathrm{Al}$ and $\mathrm{Fe} \mathrm{K}_{\alpha} \mathrm{X}$-rays in JGb-1, and $\mathrm{Mg}$ and $\mathrm{Fe} \mathrm{K}_{\alpha} \mathrm{X}$-rays in JP-1 are about $4,70,2$, and $80 \mu \mathrm{m}$, respectively.

The normalized fluorescence X-rays intensity decrease depending on the incidence angle is attributed to the above described effect for low energy X-rays, as can be seen in Fig. 11. Thus, this result of the simulations supports that trend of the relative ratios.

As shown in Figs. 8 and 11, the change on both count number relative ratio of $\# 6000$ and the normalized fluorescence X-ray intensity between $15^{\circ}$ and $30^{\circ}$ is small, whereas the change between $45^{\circ}$ and $60^{\circ}$ is larger than 0.03 in the count number relative ratio and 0.007 in the normalized fluorescence X-ray intensity at least. The depth in the sample at which the fluorescence X-rays are created depends on $\cos \theta_{i}$. Therefore, the count number relative ratios of low energy fluorescence X-rays are significantly larger for larger incidence angles and follows a non-linear. 
(a)

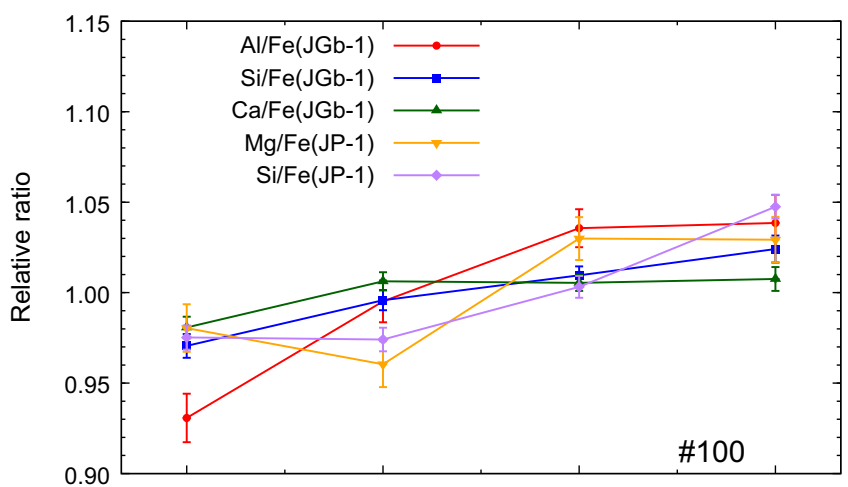

(b)

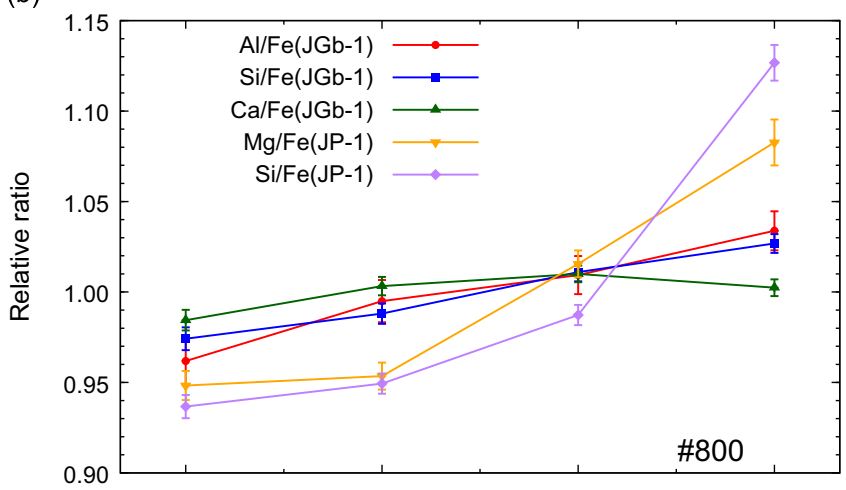

(c)

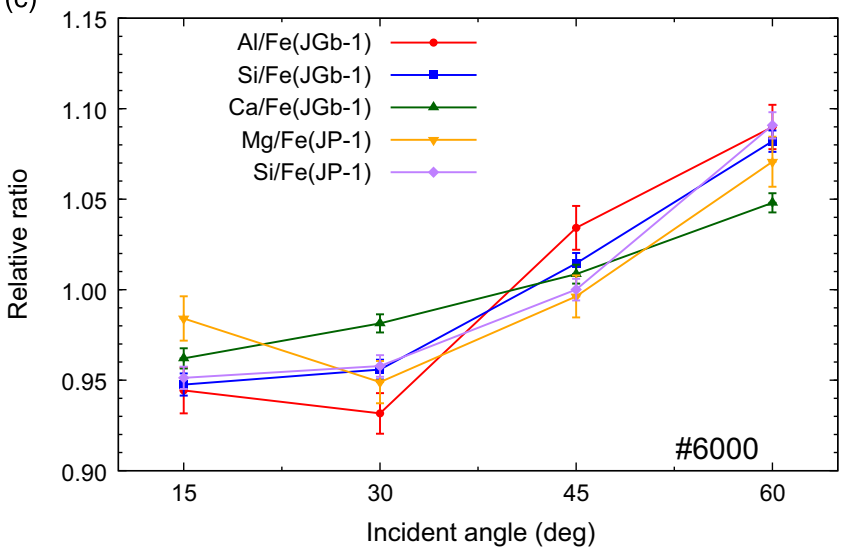

Fig. 8. The count number relative ratio as a function of the incidence angle in geometry A. Three kinds of glass samples ground by hones with \#100 (a), \#800 (b), and $\# 6000$ (c) were used.

The fluorescence X-ray creation depth is also affected by surface roughness. For rougher surface, the depth fluctuations become larger than the attenuation lengths, and that minimizes the effect described above.

\subsubsection{Geometry B}

For the roughest surface in Fig. 9 (hone \#100), the relative ratio decreased with increasing incidence angle. On the other hand, the relative ratio for the smoother surfaces is nearly constant.

In geometry $\mathrm{B}$, the emission angle is the same as the incidence angle. Note that the normalized fluorescence X-ray intensity in Fig. 11 corresponding to that of geometry B is nearly constant for all incidence angles. This is explained by the simple fact that the distance traveled by the fluorescence X-rays does not change with (a)

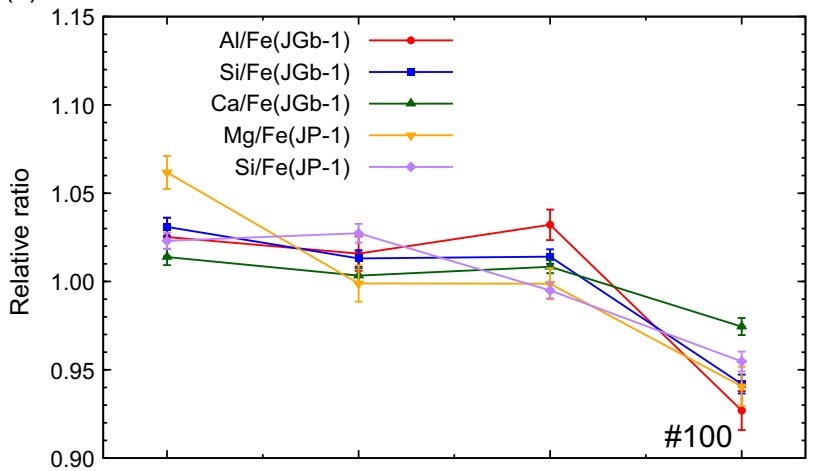

(b)

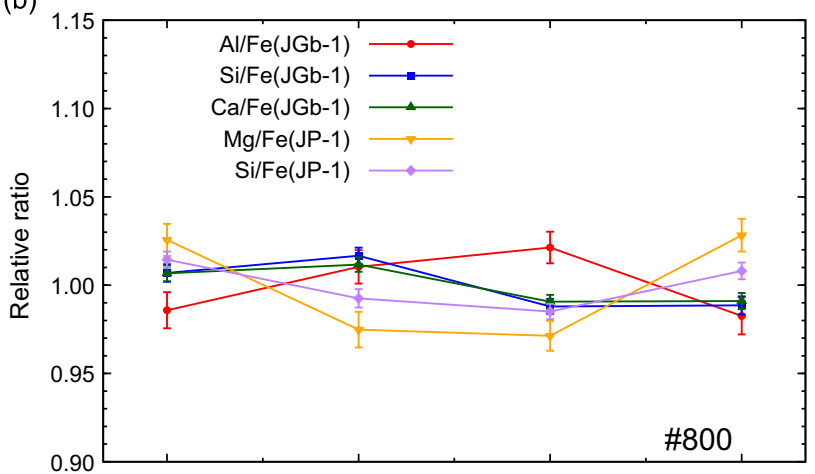

(c)

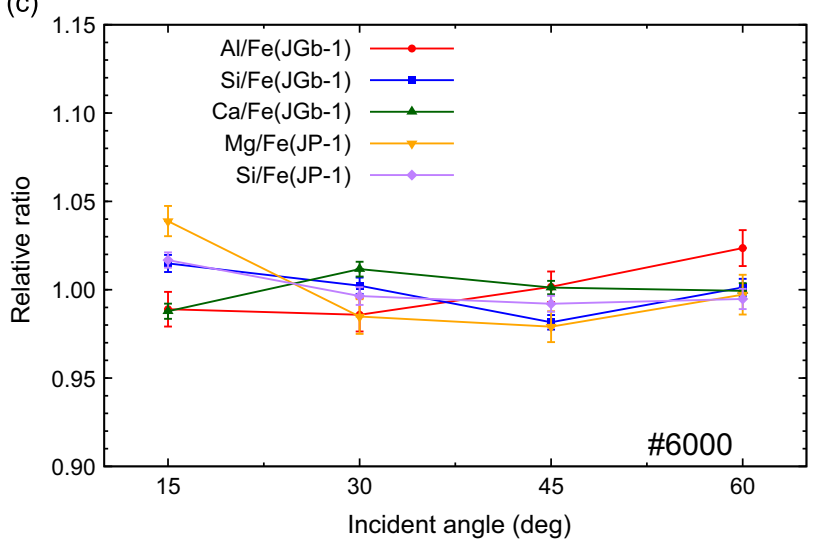

Fig. 9. The count number relative ratio as a function of the incidence angle in geometry B. Three kinds of glass samples ground by hones with \#100 (a), \#800 (b), and $\# 6000$ (c) were used.

the incidence angle in this geometry. Therefore, it is natural to expect the count number relative ratio to be nearly constant for the smoother surfaces, as can be seen in Fig. 9 (hone \#800 and 6000).

The clearer attenuation at large incidence angle for rough surface in Fig. 9 is observed for lighter elements. If we experience this level of roughness, the roughness effect becomes larger with an increase of the incidence angle. Considering the attenuation length in Section 4.2.1 and $R_{a}$ in Fig. 3, this trend is reasonable and it is considered that roughness obtained by hones with mesh number over \#800 is enough in grinding.

\section{Summary}

We have conducted XRF experimental and numerical studies to decide the AXS configuration toward the future mission. Samples 
(a)

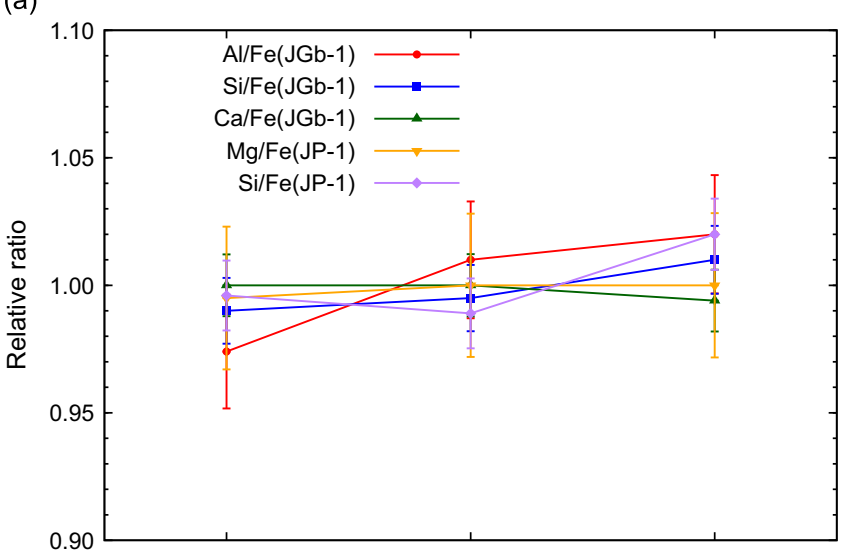

(b)

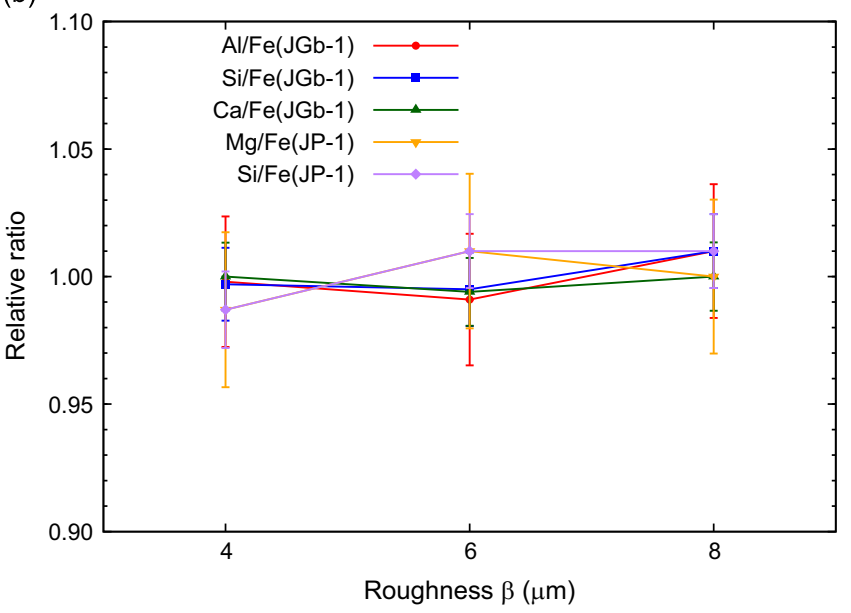

Fig. 10. The count number relative ratio obtained by simulations as a function of roughness parameter $\beta$ at $45^{\circ}$ incidence angle in (a) geometry A and (b) geometry B .

for the experiment were fused to make homogeneous glass sample to avoid influence of grain size and porosity of the sample surface. The glass samples were ground by hones with different mesh numbers to obtain different roughness and irradiated by $\mathrm{Ag} \mathrm{L}_{\alpha}$ and $\mathrm{Ag} \mathrm{L}_{\beta 1} \mathrm{X}$-rays in two different angle geometries. The simulations were also carried out following the same setup.

The largest detection efficiency was obtained for large incidence and small emission angles, especially for the lower energy fluorescence X-rays. The optimal configuration for the AXS is $\geq 45^{\circ}$ incidence angle and $0^{\circ}$ emission angle. For the fixed incidence angle of $45^{\circ}$, the effect from roughness was so small that grinding by hones of $\# 800$ is enough. The results obtained in this study are useful for future space missions employing XRF.

As was mentioned in Section 1, the effects from sample grain size and porosity as well as the less significant incident X-ray beam intensity dependence on incidence angle have not been explored.

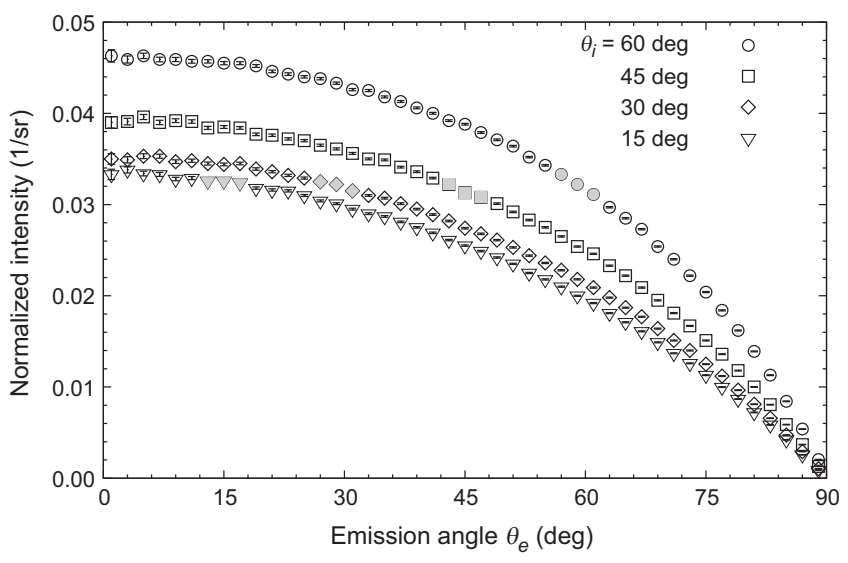

Fig. 11. Fluorescence X-ray intensity as a function of emission angle obtained by numerical simulation. Each plot represents the incidence angle, $15^{\circ}, 30^{\circ}, 45^{\circ}$, and $60^{\circ}$. The intensity corresponds to the integral spectrum of fluorescence X-rays normalized by solid angle and intensity of the incident X-ray spectrum. Gray plots represent the intensity corresponding to that of geometry $\mathrm{B} ; \theta_{i} \approx \theta_{e}$.

\section{Acknowledgement}

The authors would like to thank the Research Support Center at Waseda University for measuring the sample surface roughness.

\section{References}

[1] A.B. Binder, Science 281 (1998) 1475.

[2] S. Nozette, et al., Science 266 (1994) 1835.

[3] M. Kato, et al., Space Science Reviews 154 (2010) 3.

[4] Z. Ouyang, et al., Chinese Journal of Space Science 30 (2010) 392.

[5] P.J. Li, et al., Science China-Physics, Mechanics and Astronomy 55 (2012) 514.

[6] J.N. Goswami, M. Annadurai, Current Science 96 (2009) 486.

[7] T. Hashimoto, et al., Acta Astronautica 68 (2011) 1386.

[8] K.J. Kim, et al., Transactions of the Japan Society for Aeronautical and Space Sciences, Aerospace Technology Japan 12 (ists29) (2014) PK35.

[9] Z. Sun, et al., Science China Technological Sciences 11 (2013) 2702.

[10] B.C. Clark III, et al., Journal of Geophysical Research 82 (1977) 4577.

[11] M.P. Golombek, et al., Science 278 (1997) 1743.

[12] J.A. Crisp, et al., Journal of Geophysical Research 108 (2003) 8061.

[13] J.P. Grotzinger, et al., Science 343 (2014) 1244734

[14] J.D. Brownridge, Nature 358 (1992) 287.

[15] S.M. Shafroth, et al., Nuclear Instruments and Methods in Physics Research Section A 422 (1999) 1.

[16] H. Kusano, et al., Proceedings of SPIE 9213 (2014) 921316.

[17] Y. Maruyama, et al., Earth, Planets and Space 60 (2008) 293.

[18] Näränen, et al., Icarus 198 (2008) 408.

[19] Näränen, et al., Advances in Space Research 44 (2009) 313.

[20] S.Z. Weider, et al., Planetary and Space Science 59 (2011) 1393.

[21] H. Kusano, et al., Proceedings of SPIE 8852 (2013) 88520B.

[22] N. Imai, et al., Geostandards Newsletter 19 (1995) (1994) 135.

[23] N. Imai, et al., Geochemical Journal 29 (1995) 91.

[24] F. Salvat, et al., PENELOPE-2008: A code system for Monte Carlo simulation of electron and photon transport, in: Workshop Proceedings, NEA 6416, 2009, ISBN 978-92-64-99066-1. 